\title{
Preliminary In Vitro Effects of CD8+ T Lymphocyte Specific for the CD20 Alternative Splicing D393-CD20 Peptide Expressed on Burkitt Lymphoma Cells
}

\author{
Hamid Chegni', Zuhair M Hassan ${ }^{1 *}$, Roberto Nisini², Marzieh Ebrahimi ${ }^{3}$, \\ Farzaneh Sabouni ${ }^{4}$
}

\begin{abstract}
The effective discovery of clinically relevant tumor antigens holds a fundamental role for the development of new diagnostic tools and anticancer immunotherapies. D393-CD20 mRNA is absent from normal resting B cells but present in various malignant or transformed $\mathrm{B}$ cells. CD8+T lymphocytes play a central role in immunity to cancer. In this study, we want use from T CD8+ against D393-CD20 for effect in RAMOS cell line. After isolation and expanding of specific TCD8 + Lymphocyte against D393-CD20 antigen, for examining the effect of specialized T lymphocyte clone of D393-CD20 antigen on RAMOS cell line, we co-cultured them together, and the rate of apoptosis were examined by flow cytometry and cytotoxicity techniques by using MTT technique. We observed that specialized TCD8+ lymphocyte of D393-CD20 antigen can induce apoptosis in malignant B-lymphocytes, and this antigen can be a proper target for immunotherapy.
\end{abstract}

Keywords: CD8+ T Lymphocyte- Alternative splicing- D393-CD20 peptide- Burkitt lymphoma

Asian Pac J Cancer Prev, 20 (8), 2563-2568

\section{Introduction}

CD8+T lymphocytes play a central role in immunity to cancer through their capacity to kill malignant cells upon recognition by T-cell receptor (TCR) of specific antigenic peptides presented on the surface of target cells by human leukocyte antigen class I (HLA-I)/beta-2-microglobulin $(\beta 2 \mathrm{~m})$ complexes (Bossi et al., 2002). Major CTL activities are mediated either directly, through synaptic exocytosis of cytotoxic granules(containing perforin and granzymes) into the target, resulting in cancer cell destruction, or indirectly, through secretion of cytokines, including interferon (IFN) $\gamma$ and tumor necrosis factor (TNF) (Anikeeva et al., 2005; Le floc'h et al.,2007). Evidence for antitumor CD8+T-cell immunity was provided by isolation of tumor-specific CTL from peripheral blood or tumor tissue of patients with diverse cancers, such as melanoma and lung carcinoma (Boon et al.,1997; Echchakir et al., 2000; Karanikas, 2001; Slingluff et al.,1994; Weynants et al., 1999). The existence of a tumor-specific CTL response was further strengthened by identification of tumor associated antigens (TAA) and detection of TAA-specific $\mathrm{CD} 8+\mathrm{T}$ cells in spontaneously regressing tumors (Boon et al., 1997). Moreover, a correlation between tumor progression control and the infiltration rate of $\mathrm{CD} 8+\mathrm{T}$ lymphocytes in the tumor was established (Pages et al., 2005). The effective discovery of clinically relevant tumor antigens holds a fundamental role for the development of new diagnostic tools and anticancer immunotherapies. Generally, tumor antigens are classified as unique antigens derived from point mutations or as shared antigens (Cheever et al., 2009). One mechanism that would lead to such priority targets could be alternative splicing. Alternative splicing can change the structure of mRNA by inclusion or skipping of exons, and this may alter the function, stability or binding properties of the encoded protein (Faustino and Cooper., 2003). Aside from its role in physiological cell adaptation, alternative splicing has been shown to occur in human diseases, including cancer (Kaida et al., 2012). Particularly, the splice variants differ between cancer and normal corresponding tissues (Gardina et al., 2006; He et al., 2007). Cancer-specific splice variants are thus of significant interest as they may be involved in pathogenesis and may further potentially be used as biomarkers and generate novel targets for therapy.

CD20 protein highlighted in 1980 as a B lymphocyte specific cell-surface antigen expressed in all stages of $B$

${ }^{1}$ Department of Immunology, School of Medical Sciences, Tarbiat Modares University, ${ }^{3}$ Department of Stem Cells and Developmental Biology, Cell Science Research Centre, Royan Institute for Stem Cell Biology and Technology, ACECR, ${ }^{4}$ National Institute of Genetic Engineering and Biotechnology, Tehran, Iran, ${ }^{2}$ Department of Infectious, Parasitic and Immune-Mediated Diseases, National Institute of Health, Viale Regina Elena 299, 00161 Rome, Italy.*For Correspondence: hasan_zm@modares.ac.ir 
cell ontogenesis except for early pro-B cells and plasma cells (Argion et al., 1996) we previously identified an alternative transcript of the $\mathrm{B}$ cell lineage membrane receptor CD20. This alternative transcript lacks 168 nucleotides within Exon 3 to 7 compared to the wild-type CD20 transcript and referred thereafter as D393-CD20 (Henry et al., 2010). D393-CD20 translation gives rise to a protein lacking the extracellular domain and the most part of the four trans membrane-spanning domains and is, therefore, cytoplasmic. D393-CD20 mRNA is absent from normal resting $B$ cells but present in various malignant or transformed B cells (Henry et al., 2010; Gamonet et al., 2014; Small et al., 2013). Moreover, high expression of D393-CD20 protein has been found in malignant B cells of relapsed patients previously treated with the anti-CD20 therapeutic monoclonal antibody Rituximab (Henry et al., 2010). Therefore, we reasoned that the selective expression in leukemic B cells, as well as the expression of D393-CD20 in resistant lymphomas confers to this spliced mRNA the potential to be a tumor associated antigen. Collectively, results show that the splicing variant D393-CD20 is immunogenic and support the interest to stimulate or adoptively transfer D393-CD20-specific T cells (Vauchy et al., 2015). In this study, we want use from T CD8+ against D393-CD20 for effect in RAMOS cell line.

\section{Materials and Methods}

\section{Making D393-CD20 peptide and choosing proper solvent for it}

D393-CD20 peptide with determined amino acid sequential including KPLFRRMSSLELVIAGIVEN, was made by Bio Basic Company in Canada with $98 \%$ purification and stored in $-20^{\circ} \mathrm{C}$ in Leopligia condition. Solubility of the peptide were assessed in polar and non-polar solutions with Phi calculation, and it is determined that the peptide has high solubility in water.

Separation of D393-CD20 anti-antigens specialized T clone from normal blood sample

From normal individual (MHC CLASS I-A2) $20 \mathrm{cc}$ blood were taken, on heparin. Then separation preformed with use of PBMC ficoll (sigma). Cells were counted by using Trypan Blue staining and Hemocytometer slide, then they were cultured in RPMI (non-essential amino acids, + glutamine sodium + pyruvate + antibiotics (penicillin and streptomycin) $+5 \%$ autologous serum) in 48 wells plate in a way that in each well 2.105 number of cells perched, and we added $10 \mu \mathrm{g} / \mathrm{mL}$ of D393-CD20 peptide into each well; then we incubated the plate in $37^{\circ} \mathrm{C}$ and $5 \% \mathrm{CO}_{2}$ condition. We added 10 lambdas of IL-2 cytokine $(10 \mathrm{IU} / \mathrm{ml})$ in all of the wells after 5 days (the goal of using low dosage is that making a competitive situation for its up take with lymphocytes that could increase the expression of IL-2 receptors with recognizing intended peptide), again then we incubated the plate in $37^{\circ} \mathrm{C}$ and $5 \% \mathrm{CO}_{2}$ condition. Moreover, in 10th day we added 10 lambdas of IL-2 cytokine $(50 \mathrm{IU} / \mathrm{ml})$ into all the wells (Vauchy et al., 2014). In day 13, wells were investigated for cell growth by using invert microscope; the cell number of each well were determined by using Trypan Blue staining and Hemocytometer slide, and the wells with higher growth in cells and numbers were chosen. The contents of the chosen wells were collected in a falcon tube and then centrifuged then existing cells were placed in $1 \mathrm{cc}$ volume of RPMI including $10 \%$ FBS.

\section{Limiting Dilution}

4.105 cells from cellular suspension in previous stage were poured in $1 \mathrm{cc}$ of RPMI and we used Limiting Dilution method for separation of specialized clone (Mariotti and Nisini, 2009). After preparing proper concentrations, cells distributed in Terasaky Plate and incubated for 12 days. The wells content that have alive cells and colonial shape, transferred to wells of $96 \mathrm{U}$ bottomed wells plates and we added RPMI medium with $10 \%$ of FBS and IL-2 $(100 \mathrm{U} / \mathrm{ml})$ to them. Wells with higher growth chosen and transferred to 24-ply plate, then all the wells investigated for colony shape with inverted microscope and the wells with colony shapes were selected by using ELISA test to determining the specialty of the colony.

Determining specialty of Multiplied clones with use of ELISA technique

At first, autologous PBMC were prepared and distributed in smooth wells of 96-ply plate in a way that in each well 5.104 cells were placed. For each separated clone we considered two wells, one as positive control that D393-CD20 peptide were added to it with $10 \mu \mathrm{g} / \mathrm{mL}$ concentration, and in second group that we considered it as negative control, D393-CD20 peptide were not added. The plates were incubated in $37^{\circ} \mathrm{C}$ and $5 \%$ of $\mathrm{CO}_{2}$ for 24 hours. This period of incubation was performed, so the monocytes transformed to macrophages by attaching to the bottom of plate and they can uptake the existing D393-CD20 peptide in the culture; therefore, they can be delivered to specialized clone in next step. After 24 hours for deactivation of PBMC, we used Mitomycin C with $1 \mathrm{mg} / \mathrm{mL}$ concentration and we added Mitomycin $\mathrm{C}$ to all of the wells, then we rinsed the wells five times by using RPMI environment without FBS. The selected clones were in touch with deactivated PBMCs with Mitomycin $\mathrm{C}$ in mentioned plates for investigating their spatiality. Supernatant of were removed for evaluation of IFN $\gamma$ cytokine level. For each clone, evaluation of IFN $\gamma$, in positive (incubated by D393-CD20 peptide) and negative (incubated without D393-CD20 peptide) wells investigation preformed separately. Clones that have twice amount of IFN $\gamma$ cytokine level in positive wells vs. negative wells selected as the specialized clones for D393-CD20 (Godet et al., 2012).

\section{Proliferation of specialized D393-CD20 anti-antigen}

At first co stimulation medium environment (including Mitomycin PBMC, IL2 and PHA) was prepared (Godet et al., 2012), then half of above solution of selected wells were removed and replaced with Co stimulation medium and the wells were incubated in $37^{\circ} \mathrm{C}$ and $5 \%$ of $\mathrm{CO}_{2}$ situation. The wells were examined daily and in case of proliferation and observation of yellow color on 
the surface of media, the contexts of each wells poured in two separate wells and then we added RPMI medium including $10 \%$ of FBS to them. Every four days we added IL-2 cytokine with $50 \mathrm{IU} / \mathrm{mL}$ concentration to all of the wells. Since we needed high number of D393-CD20 specialized anti-antigen cells for in vitro studies, this method of proliferation was continued for 50 days (Nikolova et al., 2003).

Analyzing the specialized T lymphocyte clone of D393-CD20 antigen on RAMOS cell line

RAMOS cell line was taken from cell bank of Pasteur Institute of Tehran and after transporting to laboratory for the study, they were sub-cultured. For examining the effect of specialized T lymphocyte clone of D393-CD20 antigen on RAMOS cell line, we co-cultured them together, and the rate of apoptosis were examined by flow cytometry and cytotoxicity techniques by using MTT technique.

\section{Results}

After expose of separated clones with D393-CD20 peptide and comparison of them with self-control (without neighboring with the peptide), the amount of IFN in above solution of culture were studied, and the clones that have significant differences after expose with peptide (positive control) and without peptide (negative control) were selected, among 20 studied clones, the clone number 5 had the most discrepancy in level of IFN production in adjacency to the peptide in comparison with its negative control group. In addition, clones number 11, 15 and 6 also have significant differences in their amount of IFN production.

The results of analyzing features of selected T lymphocyte clones with flow cytometry technique

After staining some of the selected T lymphocyte clone cells of D393-CD20 antigen with Anti CD4 connected to FITC stain and Anti CD8 connected to PE, by using flow cytometry device, it was determined that $86.6 \%$ of selected cell populations were CD8+ and CD4-.

Result of analyzing RAMOS cell line apoptosis by using flow cytometry technique

In U shape wells of 96-ply plate, we co-cultured the selected specialized clones (clone number 5, 11, 15, 6) with RAMOS cell line in ratio of 1 to 10 . We co-cultured one of the negative clone in selection phase with RAMOS cell line as negative control. The results were obtained

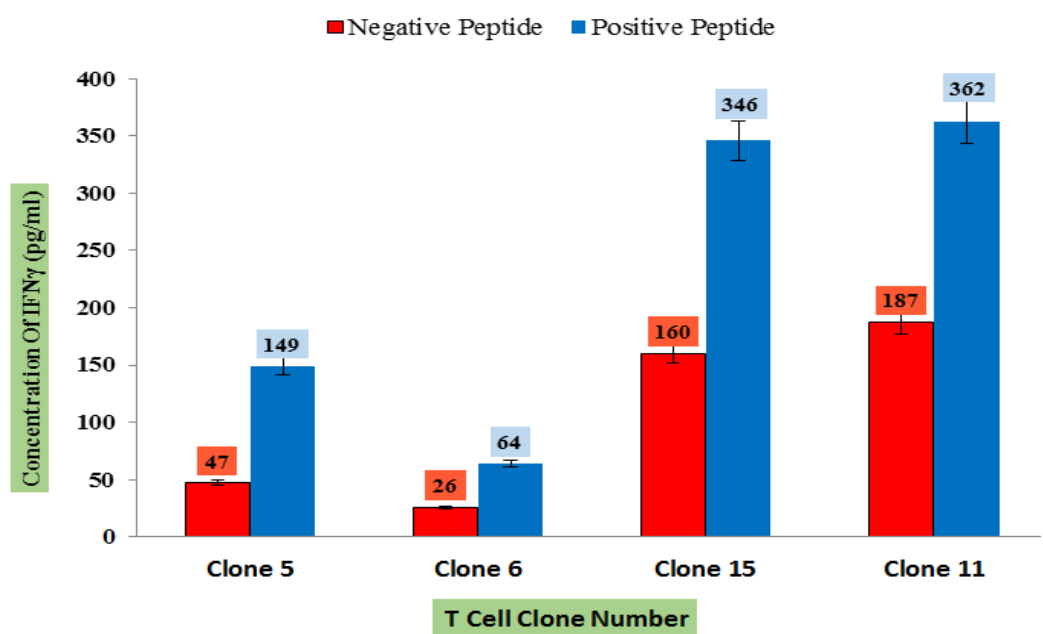

Diagram 1. Comparison of the Amount of IFN $\gamma$ Secretion of Selected Clones in Juxta Positioning Situation with D393-CD20 Peptide (Positive Peptide) and without D393-CD20 Peptide (Negative Peptide).

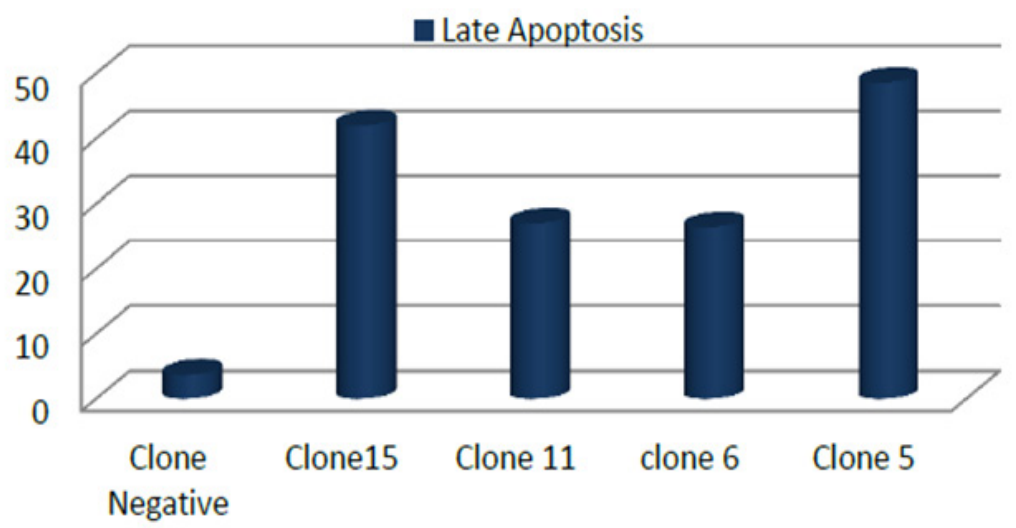

Diagram 2. The Results from Percentage of RAMOS Cell Line Apoptosis in Co-culture with Specialized T Lymphocyte Clones of D393-CD20 Antigen and Its Comparison with Unspecialized T Lymphocyte Clones of D393-CD20 Antigen as Negative Control with Apoptosis Technique. 
Table 1. The Results of MTT Test Related to RAMOS Cell Line Co-culture with Specialized T Lymphocyte Clones of D393-CD20 Antigen and Non-specialized Clone for Negative Control

\begin{tabular}{lcccc}
\hline Clone number & RAMOS cell line OD & T lymphocyte clone OD & Co-culture OD & Cytotoxicity Percentage \\
\hline Negative CLONE & 0.212 & 0.199 & 0.393 & 4.37 \\
CLONE 5 & 0.236 & 0.262 & 0.177 & 64.45 \\
CLONE 15 & 0.212 & 0.185 & 0.194 & 51.13 \\
CLONE 11 & 0.212 & 0.173 & 0.271 & 26.61 \\
CLONE 6 & 0.128 & 0.148 & 0.194 & 29.71 \\
\hline
\end{tabular}

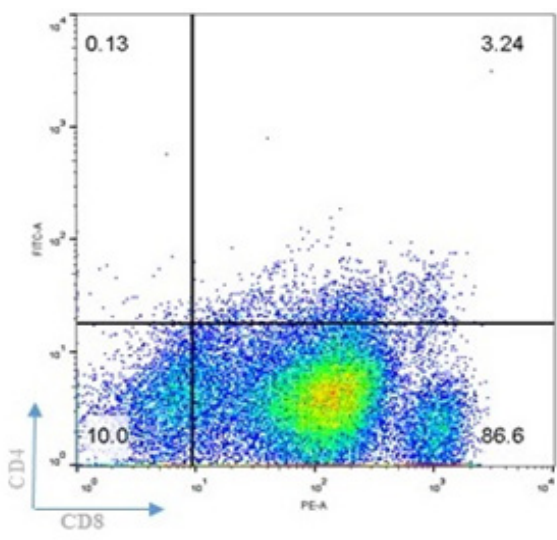

Figure 1. Results from Flow Cytometry Regarding to Selected T Lymphocytes of D393-CD20. Antigen with Anti CD4 connected to FITC stain and Anti CD8 connected with PE, that shows $86.6 \%$ of the cells are CD4- and CD8+.

by flow cytometry device and after analyzing the data, it was determined that clone number 5 induced the most apoptosis in RAMOS cell line.
Results of MTT test related to RAMOS cell line co-cultured with specialized T lymphocytes of D393-CD20 antigen

We placed the plate in ELISA reader device and we measured the amount of absorption in $450 \mathrm{~nm}$ wavelength. For each clones of T lymphocytes, three types of wells were chosen as follows: one well including T lymphocyte clone without RAMOS cell line, one well including RAMOS cell line without $\mathrm{T}$ lymphocyte clone and one well including co-culture of $\mathrm{T}$ lymphocytes clone with RAMOS cell line.

The results of MTT test were calculating with this formula:

The percentage of immolated cells $=(($ RAMOS cell line OD + T lymphocyte)OD- CO culture OD)/((RAMOS cell line OD+ T lymphocyte OD))

After comparing the percentage of RAMOS cell line in co-culture with specialized TCD8+ lymphocyte clones of D393-CD20 antigen, which was selected in previous stage, it was determined that clones number 5 and 15 had the rate of 64.45 and 51.1 for induction of cytotoxicity in co-culturing with RAMOS cell line, respectively. These results are in agreement with the results from flow cytometry.
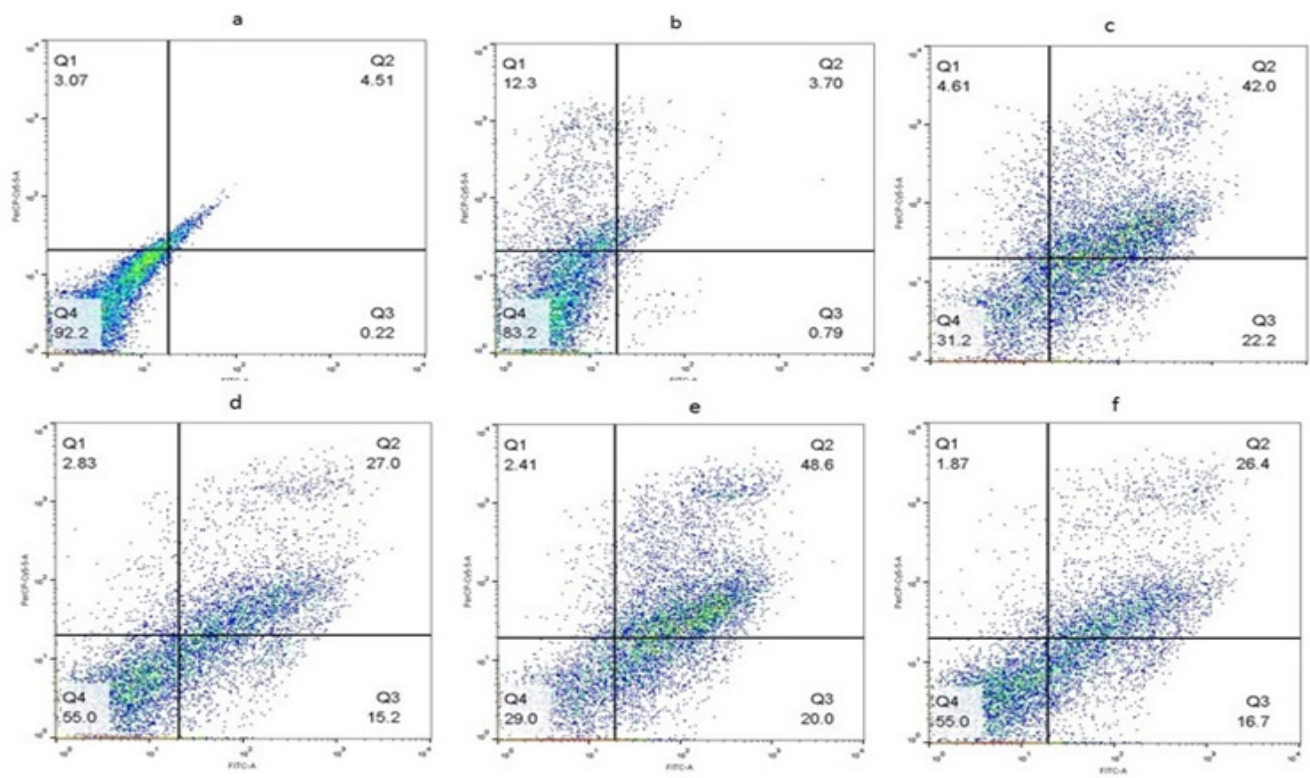

Figure 2. Result of Analyzing RAMOS Cell Line Apoptosis by Using Flow Cytometry Technique. a) RAMOS cell line alone (as control). b) Cocuture of RAMOS cell line and Negative Control (CD8+ T clones nonspecific for D393-CD20 peptide). c) Cocuture of RAMOS cell line and CD8+ T clones specific for D393-CD20 number 15.d) Cocuture of RAMOS cell line and CD8+ T clones specific for D393-CD20 number 6. e) Cocuture of RAMOS cell line and CD8+ T clones specific for D393-CD20 number 5.f) Cocuture of RAMOS cell line and CD8+ T clones specific for D393-CD20 number 11. 


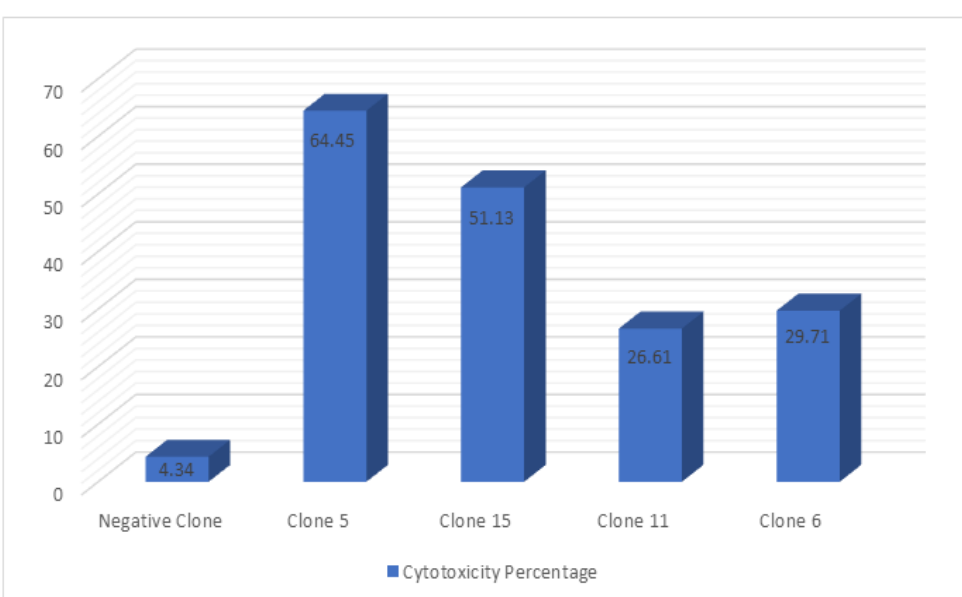

Diagram 3. The Results from RAMOS Cell Line Cytotoxicity Percentage in Co-Culture with Specialized T Lymphocyte Clones of D393-CD20 Antigen and Its Comparison with non-Specialized T Lymphocyte Clone of D393-CD20 Antigen as Negative Control with MTT Technique.

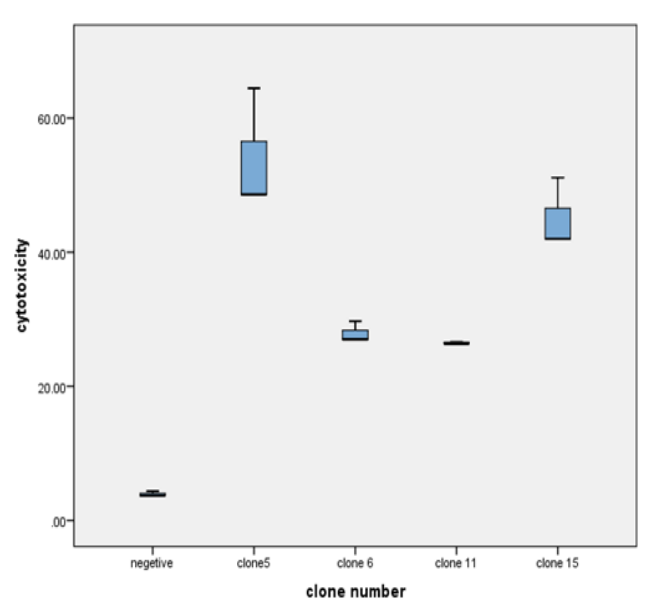

Figure 3. In Vitro Cytotoxicities RAMOS Cell Line Co-culture with Specialized T Lymphocyte Clones of D393-CD20 Antigen and Non-specialized Clone for Negative Control $(n=10)$ Means \pm SDs, $* p<0.5$

\section{Discussion}

Recent studies showed that alternative splicing would produce different types of tumor antigen (Zhang et al., 2013; Lin et al., 2004). Alternative splicing can alter mRNA structure by adding or eliminating exons and it can affect the function, sustainability, and connectional features of proteins (Faustino and Cooper, 2003). The tumor specialized created variants can affect the pathogenesis of tumor and they can be used as specific biomarkers for new diagnostic and treatment methods. In a study by Kobayashi et al., (2009) in Japan on 4 patient suffering from mouth cancer, systemic blood lymphocytes of patients in vitro adjacent with peptides of Survivin sub-types. Result showed that peptides related to Survivin-B80-88 had the highest immunogenicity for CTL, so they can be good candidates for immunotherapy against Survivin in digestive, colon, breast, lung, bladder and mouth tumors. Recently, a spliced mRNA transcript of CD20 (named D393-CD20) was identified in malignant B cells from lymphoma and leukemia patients. D393-CD20 lacks 168 nucleotides within Exon 3 to 7 compared to the wild-type CD20 transcript. D393-CD20 $m R N A$ is absent from normal resting $\mathrm{B}$ cells but present in various malignant or transformed B cells (Henry et al., 2010; Gamonet et al., 2014; Small et al., 2013).

Other study which performed by Vauchy et al., (2015), determined that D393-CD20 antigen exists in surface of malignant $\mathrm{B}$ cells and also CD4+ T cells specific for D393-CD20 are detectable in B cell lymphoma patients and can produce IFN. In addition performed vaccination with D393-CD20 antigen peptides in human HLA transgenic mice model and sowed that D393-CD20 antigen peptides may bind both HLA classes I and class II MHC molecules, so that both TCD8+ and TCD4+ can be expanded. This study proposed that targeting D393-CD20 antigen could perform more effective immunotherapy in treatment of B cell malignant (Vauchy et al., 2014). In another study, Gamonet et al., (2015) determined that D393-CD20 antigen exist in surface of B cells tumor and that the EBV transformation of B cells can increase the membrane expression of D393-CD20. In addition, in case of CLL appeared that the rate of D393-CD20 expression in surface of tumor cells is higher in patients that are in B and C stages. Benjamin (2014) determined that anti-CD20 monoclonal antibody (Rituximab) could be effective in treatment of B-NHL tumor. Using this therapeutic approach along with chemotherapy, radiotherapy and proteasome inhibitors can lead to more effectiveness in treatment. Some patients have been observed to became tolerant to Rituximab for still unclear reason (s). However, the level of D393-CD20 expression increased also in tolerant patients. According the recent findings, D393-CD20 can represent a good candidate for immunotherapy due to its high immunogenicity and its expression in malignant B -lymphocytes but not on normal cells.

While the function of D393-CD20 remains, obscure (Henry et al., 2010). Moreover, there are very few studies concerning D393-CD20 peptide, in this study, we determined that CD8+ T clones specific for D393-CD20 peptide can directly recognize the $\mathrm{B}$ cells tumors and induce their apoptosis. The capacity of CD8+ T cells to directly recognize the malignant cells confirms previous 
papers showing that D393-CD20 is a naturally processed antigen in tumor B cells that may bind nascent $\mathrm{MHC}$ class I molecules migrating to the tumor cells membrane.

As a summary of our study, we isolated a series of D393-CD20 specific CD8+ T lymphocyte clones from PBMC of a normal individual. These clones in vitro proliferated in the presence of synthetic D393-CD20 peptide and in the presence of the B cell line RAMOS. From a functional point of view, our clones induced apoptosis of the malignant B cell line RAMOS, observed by flow cytometry, as well as its killing, as observed by the MTT method. The capacity of D393-CD20 to in vitro induce expansion of specific CD8+ T lymphocytes with killing activity against malignant cells naturally expressing the antigen, suggest that this antigen can be a proper target for immunotherapy. Although this study represents a preclinical approach to $\mathrm{T}$ cell transfer therapy and further in vivo studies in animal models are required, it suggest that D393-CD20 is highly immunogenic also for CD8+ T cells and that it can be exploited for future therapeutic approaches.

\section{References}

Algino KM, Thomason RW, King DE, Montiel MM, Craig FE (1996). CD20 (pan-B cell antigen) expression on bone marrow-derived T cells. Am J Clin Pathol, 106, 78-81.

Anikeeva N, Somersalo K, Sims TN, et al (2005). Distinct role of lymphocyte function-associated antigen-1 in mediating effective cytolytic activity by cytotoxic T lymphocytes. Proc Natl Acad Sci U S A, 102, 6437-42.

Boon T, Coulie PG, Van den Eynde B (1997). Tumor antigens recognized by T cells. Immunol Today, 18, 267-8.

Bossi G, Trambas C, Booth S, et al (2002). The secretory synapse: the secrets of a serial killer. Immunol Rev, 189, 152-60.

Benjamin B (2014). Postulated mechanisms of resistance of B-cell non-hodgkin lymphoma to rituximab treatment regimens: Strategies to overcome resistance. Seminoncol, 41, 667-77.

Cheever MA, Allison JP, Ferris AS, et al (2009). The prioritization of cancer antigens: a national cancer institute pilot project for the acceleration of translational research. Clin Cancer Res, 15, 5323-37.

Echchakir H, Vergnon I, Dorothee G, et al (2000). Evidence for in situ expansion of diverse antitumor-specific cytotoxic $\mathrm{T}$ lymphocyte clones in a human large cell carcinoma of the lung. Int Immunol, 12, 537-46.

Faustino NA, Cooper TA(2003). Pre-mRNA splicing and human disease. Genes Dev, 17, 419-37.

Gardina PJ, Clark TA, Shimada B, et al (2006). Alternative splicing and differential gene expression in colon cancer detected by a whole genome exonarray. BMC Genomics, 7, 325-46.

Godet Y, Fabre E, Dosset M, et al (2012).Analysis of spontaneous tumor-specific CD4 T-cell immunity in lung cancer using promiscuousHLA-DR telomerase-derived epitopes: potentialsynergistic effect with chemotherapy response. Clin Cancer Res off J Am Assoc Cancer Res, 18, 2943-53.

Gamonet C, Ferrand C, Colliou N, et al (2014). Lack of expression of an alternative CD20 transcript variant in circulating B cells from patients with pemphigus. Exp Dermatol, 23, 66-7.

Gamonet C, Bole-Richard E, Delherme A, et al (2016). New CD20 alternative splice variants: molecular identification and differential expression within hematological B cell malignancies. Bio Med Central, 366, 219-27.

He C, Zuo Z, Chen H, et al (2007). Genome-wide detection of testis- and testicular cancer-specific alternative splicing. Carcinogenesis, 28, 2484-90.

Henry C, Deschamps M, Rohrlich P-S, et al (2010).Identification of an alternative CD20 transcriptvariant in B-cell malignancies coding for a novelprotein associated to rituximab resistance. Blood, 115, 2420-9.

Karanikas V, Colau D, Baurain JF, et al (2001). High frequency of cytolytic T lymphocytes directed against a tumor-specific mutated antigen detectable with HLA tetramers in the blood of a lung carcinoma patient with long survival. Cancer Res, 61, 3718-24.

Kobayashi J, Torigoe T, Hirohashi Y, et al (2009). Comparative study on the immunogenicity between an HLA-A24-restricted cytotoxic T-cellepitope derived from survivin and that from its splice variant survivin-2B in oral cancer patients. J Transl Med, 7, 1.

Kaida D, Schneider-Poetsch T, Yoshida M (2012). Splicing in oncogenesis and tumor suppression. Cancer Sci, 103, 1611-6.

Lin J, Lin L, Thomas DG, et al (2004). Melanoma-associated antigens in esophageal adenocarcinoma: identification of novel MAGE-A10 splice variants. Clin Cancer Res off $J$ Am Assoc Cancer Res, 10, 5708-16.

Le floc'h A, Jalil A, Vergnon I, et al (2007). Alpha E beta 7-integrin interaction with E-cadherin promotes antitu- mor CTL activity by triggering lytic granule polarization and exocytosis. J Exp Med, 204, 559-70.

Mariotti S, Nisini R (2009). Generation of human T cell clones. Methods Mol Biol, 514, 65-93.

Nikolova M, Echchakir H, Wechsler J, Boumsell L, Bensussan A (2003). Isolation of a CD8alphaalpha+ CD4- tumour T-cell clone with cytotoxic activity from a CD4+ CD8- cutaneous T-cell lymphoma. Br J Dermatol, 148, 24-9.

Pages F, Berger A, Camus M, et al (2005). Effector memory T cells, early metastasis, and survival in colorectal cancer. $N$ Engl J Med, 353, 2654-66.

Slingluff CL Jr, Cox AL, Stover JM Jr, et al (1994). Cytotoxic T-lymphocyte response to autologous human squamous cell cancer of the lung: epitope reconstitution with peptides extracted from HLA-Aw68. Cancer Res, 54, 2731-7.

Small GW, McLeod HL, Richards KL (2013). Analysis of innate and acquired resistance to anti-CD20 antibodies in malignant and nonmalignant B. Cells Peer J, 1, e31.

Vauchy C, Gamonet C, Ferrand C, et al (2014). CD20 alternative splicing isoform generates immunogenic CD4helper T epitopes. Int $J$ Cancer, 137, 116-26.

Vauchy CH, Gammoned C, Ferrand CH, et al (2015). CD20 alternative splicing isoform generates immunogenic CD4helper T epitopes. Int $J$ Cancer, 137, 116-26.

Weynants P, Thonnard J, Marchand M, et al (1999). Derivation of tumor-specific cytolytic T-cell clones from two lung cancer patients with long survival. Am J Respir Crit Care Med, 159, 55- 62 .

Zhang L, Vlad A, Milcarek C, Finn OJ (2013). Human mucin MUC1 RNA undergoes different types of alternative splicing resulting in multiple isoforms. Cancer Immunol Immunother CII, 62, 423-35.

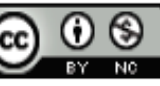

This work is licensed under a Creative Commons AttributionNon Commercial 4.0 International License. 